\title{
Aktives Leben schützt vor Demenz
}

\author{
Sport, körperliche Ertüchtigung und Alltagsaktivität schieben das Einsetzen einer Demenz \\ auf. Das zeigt eine große Zwillingsstudie aus Finnland. Sie lässt den Schluss zu, dass körperliche \\ Aktivität umso besser vor Demenz schützt, je intensiver sie ist.
}

_ Körperliche Aktivität ist geeignet, um Risikofaktoren für alle Erkrankungsentitäten des metabolischen Syndroms und auch Depressionen zu verringern. Aber wie sieht es bei Demenz aus? Leibesertüchtigung hat diverse physiologische Effekte, die auch hier positiv zu Buche schlagen könnten - man denke z.B. an die Verbesserung der Insulinresistenz, des Eiweißstoffwechsels und des Sekretionsprofils von Adipokinen und Hepatokinen oder an die Reduktion chronischer Entzündungsprozesse.

Grundsätzlich könnte man also erwarten, dass auch demenzielle Prozesse mit Ertüchtigung verhindert werden können. Dieser Zusammenhang ist aber empirisch spekulativ und konnte bisher nicht wirklich belegt werden. Es ist ja auch schwierig, eine entsprechende Studie durchzuführen, bei der man nach der Manifestation einer Demenz abwartet. Eine finnische Forschergruppe hat sich diesen Fragen aber gestellt.
Die Wissenschaftler untersuchten dafür bei Zwillingen, inwieweit sich kontinuierliche körperliche Aktivitäten im Erwachsenenalter auf die Mortalität durch Demenz auswirken. Zwischen 1975 und 1981 wurde von den Mitgliedern der Older Finnish Twin Cohort $(\mathrm{n}=21.791)$, die damals zwischen 24 und 60 Jahre alt waren, das Ausmaß körperlicher Aktivität gemessen. Die Teilnehmer wurden hinsichtlich der Intensität und der Dauer in drei Kategorien eingeteilt. Demenzdiagnosen und demenzassoziierte Todesfälle wurden bis Ende 2011 erfasst.

353 Teilnehmer aus der Zwillingskohorte starben aufgrund von Demenz. Das Risiko hierfür war in der Gruppe derer, die zwischen 1975 und 1981 körperlich aktiver waren, um 35\% niedriger als in den körperlich inaktiven Gruppen. Der Effekt wurde nach Berücksichtigung von potenziellen Störfaktoren schwächer - allerdings verstärkte er sich deutlich, wenn man ein Zwillingspaar isoliert betrachtete. Hier zeigte sich, dass körperliche Aktivität das Demenzrisiko um $52 \%$ senken kann.

- Iso-Markku P, Waller K, Kujala UM, Kaprio J. Physical activity and dementia: long-term follow-up study of adult twins. Ann Med. 2015;47:81-7

\section{KOMMENTAR}

Das ist toll! Die positiven Auswirkungen körperlicher Aktivität auf Diabetesrisiko, Arteriosklerose, Herzinfarktrisiko, Dyslipidämie und Depression sind bereits bekannt. Nun folgt eine große prospektive Untersuchung hinsichtlich des Auftretens einer Demenz. Der Effekt ist eindeutig: Das Risiko, an Demenz zu erkranken, kann durch körperliche Aktivität halbiert werden. Man mag das für wenig überraschend halten, aber die Quintessenz ist stark: Mit 10.000 Schritten am Tag läuft man nicht nur dem Diabetes, dem metabolischen Syndrom und der Depression davon kann, sondern auch der Demenz

Prof. Dr. med. P. Schwarz

\section{Malignes Melanom der Bindehaut}

Eine 78-jährige Frau mit Glaukom, Diabetes mellitus und Katarakt stellte sich routinemäßig zu einer augenärztlichen Untersuchung vor. Dabei stellte man eine rötlich-braune makulopapuläre Läsion am rechten Auge fest. Der Patientin war diese Veränderung seit etwa einem Jahr bewusst. Die Ausdehnung des Herdes hatte zu- und abgenommen, gelegentlich geblutet, war aber weder schmerzhaft noch hatte sie den Visus beeinträchtigt. Man führte eine sklerale Keratotomie mit anschließender Kryotherapie und Alkoholapplikation durch. Die histologische Untersuchung des Gewebes ergab ein malignes Melanom in Verbindung mit einer primär erworbenen Melanosis.

Konjunktivale Melanome können durch eine primär erworbene Melanosis entstehen und sind häufig mit regulären Metastasen und lokalen Rezidiven belastet. Die Patientin wurde mit Chemotherapie und topischer Anwendung von Mitomycin behandelt. Die Untersuchung der regulären Lymphknoten, ein Knochenszintigramm und eine CT von Kopf, Hals, Brust und Abdomen ergaben keinen Hinweis auf eine Metastasierung. Auch 18 Monate nach Abschluss der Therapie zeigte sich kein Rezidiv.

- Prof. Dr. med. H. S. FüeßI

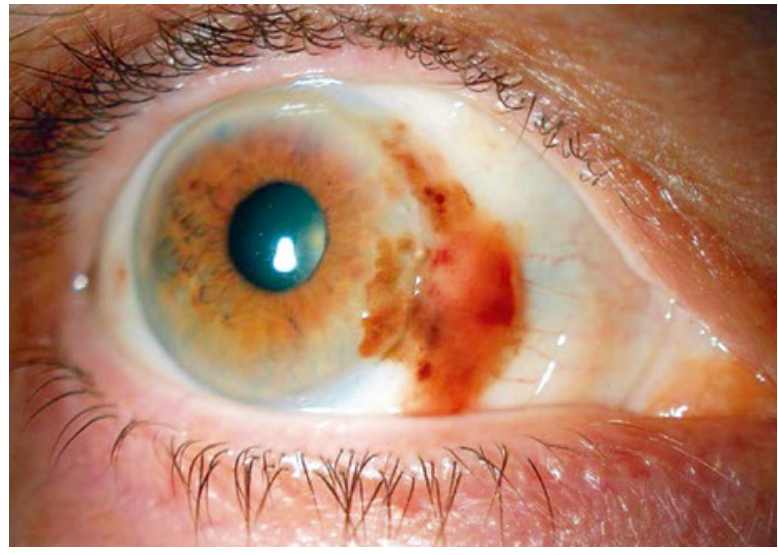

Rötlich-braune, gelegentlich blutende makulopapuläre Läsion am rechten Auge. 